\section{GP162 THE ANALGESIC EFFICACY OF ULTRASOUND-GUIDED QUDRATUS LUMBORUM BLOCK ON POSTOPERATIVE PAIN AND MORPHINE CONSUMPTION IN CHILDREN WITH APPENDICITIS}

Dmytro Dmytriiev, Bohdan Zaletskyi, Oleksandr Katilov*, Kateryna Dmytriieva. Vinnitsa national medical university, Vinnitsa, Ukraine

10.1136/archdischild-2019-epa.225

Objectives To evaluate the analgesic effect of qudratus lumborum block (QLB) in pediatric patients administered before appendicitis.

Methods This study was completed at the Faculty of Medicine, Vinnitsa national medical university, Vinnitsa, Ukraine, between September 2018 and Febrary 2019. In a prospective, double blind, randomized, placebo controlled clinical study, 38 pediatric patients scheduled for appendicitis operations were randomized to group $\mathrm{Q}$ (treatment group) or group $\mathrm{C}$ (controls). After receiving general anesthesia, group $\mathrm{T}$ received a QLB block using $0,3 \mathrm{~mL}$ per $\mathrm{kg} 0.25 \%$ bupivacaine on the right side, where as group $\mathrm{C}$ received a control block using $0,3 \mathrm{~mL}$ per $\mathrm{kg} 0.9 \%$ Sodium chloride. During the first 24 hours after surgery, the patient pain was evaluated using the visual analogue scale (VAS) at rest and while coughing. Postoperative patient controlled analgesia morphine consumption, VAS scores, and side effects were recorded.

Results Of 24 patients, Group Q $(n=14)$ had significantly lower VAS pain scores than Group C $(n=10)$ both at rest and while coughing. The total morphine consumed was lower $(5.2$ \pm 2.4 ) versus $17.3 \pm 3.4 \mathrm{mg}, \mathrm{p}<0.001)$ in the 24 hours after surgery.

Conclusion As part of a multimodal analgesic regime after appendicitis, morphine consumption and VAS pain scores were significantly lower among those receiving $0,3 \mathrm{~mL}$ per $\mathrm{kg}$ $0.25 \%$ bupivacaine administered for a QL block than among controls.

\section{GP163 IMPROVING PATIENT EXPERIENCE THROUGH THE USE OF A SHORT STAY OBSERVATION UNIT IN A PEDIATRIC HOSPITAL, A PILOT STUDY}

Claire Fagan, Sharon Ryan, Elaine Fitzgerald, Roisin McNamara, Patrick Fitzpatrick, Ike Okafor, Helen Flynn*, Joan Broderick, Nandini Kandamany. CH@Temple street, Dublin, Ireland

\subsection{6/archdischild-2019-epa.226}

The Emergency Department (ED) at Children's University Hospital, Temple Street is constantly running at maximum capacity due to limited space and in-patient beds, as well as inadequate staffing. This leads to a number of issues including: reduced patient flow, department overcrowding, unacceptable patient experience times and possible adverse events. A short stay observation unit (SSOU) was introduced to improve flow and patient experience. The RCPCH (2017) have defined a short stay unit as a 'dedicated facility providing assessment, observation and treatment of an illness, without the need for inpatient admission and can be a safe and efficient way of managing their care.'

Our aim was to utilize beds in a different way to improve patient safety and patient flow in the ED, therefore improving PET times and patient satisfaction. We developed a local implementation group that met weekly to progress and change as necessary. We developed set admission criteria and standard operating procedures for the department.

Weekly audits for patient length of stay in the SSOU, ED trolley wait times monitored weekly, parent and staff satisfaction surveys, PET times monitored weekly. Over the duration of the 8 week pilot study;

Average length of stay in the SSOU was 11.7 hours

164 patients through the SSOU in 8 weeks, at 1.2 patients per available bed/day

Trolley wait times have dropped to the previous 2 years

Satisfaction surveys showed that the SSOU positively affected patients hospital experience and the ED staffs workload

The utilisation of beds and resources in a different way can have a positive impact on patient care. Parent satisfaction and patient flow can be greatly improved from implementing a permanent SSOU. The ED can be used more efficiently and patient safety improved through effective use of resources. Welcomed changes can have a big impact on staff morale.

We recommend that for future use, the SSOU be run as a standalone unit by the ED staff on a daily basis for the entirety of its opening hours. The SSOU should be in operation for at minimum the busy winter period, that being from late September to early May.

\section{GP164 REAL-TIME SURVEILLANCE OF PEDIATRIC INFECTIOUS DISEASES IN FRENCH AMBULATORY CARE}

${ }^{1}$ Stéphane Béchet* ${ }^{*}{ }^{1}$ Corinne Levy, ${ }^{2}$ François Vie Le Sage, ${ }^{2}$ Andreas Werner, ${ }^{2}$ Nathalie Gelbert, ${ }^{2}$ Georges Thiebault, ${ }^{1}$ Robert Cohen. ${ }^{1}$ ACTIV, Saint Maur, France; ${ }^{2}$ AFPA, Talence, France

\subsection{6/archdischild-2019-epa.227}

Background Infectious diseases account for 50-70\% of ambulatory pediatric daily practice. In order to improve the diagnostic performance of primary-care pediatricians by providing real time data on epidemiology of several infectious diseases, we have set up a national surveillance network, PARI (Pediatric and Ambulatory Research in Infectious diseases). This network, based on automated data extraction from the primarycare pediatricians' computers, differs from existing networks in the world by one major characteristic: the specific trainings (face-to-face meetings and e-learning website) of participating pediatricians in diagnostic and treatment of infectious diseases and the use of rapid diagnostic tests.

Methods We daily prospectively collect anonymized data (age, sex, height, weight, daycare attendance, vaccines, diagnosis and prescriptions) of children with infectious diseases, in 82 primary-care pediatricians of the French Ambulatory Pediatric Association (AFPA) using the same software (Axi5-Infansoft ${ }^{\circledR}$, CompuGroup Medical) exercising all over France. Each time a pediatrician closes the patient's file on his software, all the information concerning the infectious episode is sent to a database stored on approved and secured servers. No additional data than those required in the daily practice of the pediatricians are requested.

Results Between July 2017 and December 2018, data on 25,923 patients, 37,033 consultations, 51,568 diagnoses, 176,331 vaccines and 161,654 drug-prescriptions were collected. Mean age at infectious diagnosis was $3.0 \pm 2.9$ years and boys accounted for $57.1 \%$. Frequencies of the different infectious diseases were weekly and automatically provided on a dedicated website as graphs for all pediatricians, to allow 